\title{
Intoxication à la strychnine : un cas fatal
}

\author{
Strychnine intoxication : a fatal case
}

\section{Charlotte DUVERNEUIL ${ }^{(1)}$, Geoffroy LORIN DE LA GRANDMAISON ${ }^{(2)}$, Philippe DE MAZANCOURT ${ }^{(1)}$, Jean-Claude ALVAREZ ${ }^{(1) *}$}

(1) Laboratoire de Pharmacologie - Toxicologie

(2) Service de Médecine Légale, Centre Hospitalier Universitaire Raymond Poincaré, AP-HP,

104, Boulevard R. Poincaré - 92380 GARCHES

* Auteur à qui adresser la correspondance : Jean-Claude ALVAREZ, Laboratoire de Pharmacologie - Toxicologie, Centre Hospitalier Universitaire Raymond Poincaré, 104 Boulevard R. Poincaré - 92380 GARCHES

Tél : 0147107938 - Fax : 0147107923

Ce travail a été présenté au XI ${ }^{\mathrm{m} e}$ congrès de la Société Française de Toxicologie Analytique, 11-13 juin 2003, DINARD-FRANCE

(Reçu le 28 octobre 2003 ; accepté le 6 janvier 2004)

\section{$\boldsymbol{R E S U M E ́}$}

Un cas de décès à la strychnine, pour lequel nous ne possédions qu'un faible prélèvement de sang, a entraîné la mise au point d'une technique de dosage par chromatographie liquide haute performance couplée à une détection par barrette de diodes ne nécessitant que $0,1 \mathrm{ml}$ d'échantillon. Cette technique a également été appliquée à un échantillon de liquide gastrique et au liquide contenu dans une cannette de bière trouvée à côté du corps. La strychnine a été isolée à partir de ces échantillons par une technique d'extraction liquide-liquide, avec la chloroquine comme étalon interne. Les limites de détection et de quantification sont respectivement de $0,06 \mathrm{mg} / \mathrm{l}$ et $1 \mathrm{mg} / \mathrm{l}$. Le rendement d'extraction est de $94 \%$ et les coefficients de variation sont compris entre 5,9 et $10,8 \%$. La concentration de strychnine observée dans le sang $(25 \mathrm{mg} / \mathrm{l})$ était mortelle.

\section{MOTS-CLÉS}

Strychnine, CLHP/BD, intoxication létale.

\section{SUMMARY}

A fatal case of strychnine poisoning, with very small blood sample available, led us to develop a sensitive method which use only $0,1 \mathrm{ml}$ of sample. This method used high performance liquid chromatography coupled with photodiodearray detection. This analytical method has been applied to biological samples (blood and gastric liquid) and to a bottle of beer founded near the deceased patient. The strychnine was isolated from samples using liquid-liquid extraction procedure and chloroquine as internal standard.

The limits of detection and quantification are $0,06 \mathrm{mg} / \mathrm{l}$ and $1 \mathrm{mg} / \mathrm{l}$ respectively. The recovery is $94 \%$ and the coefficients of variation ranged from 5.9 to $10.8 \%$. The blood concentration of strychnine $(25 \mathrm{mg} / \mathrm{l})$ was lethal.

KEY-WORDS

Strychnine, HPLC/DAD, lethal intoxication. 


\section{Introduction}

La strychnine est un alcaloïde extrait à partir de la graine d'un arbre indien nommé Strychnos nux vomica. Son poids moléculaire est de 334 . Son spectre de masse et sa formule chimique sont présentés en figure 1. Le métabolisme de la strychnine est peu documenté. Elle est rapidement absorbée au niveau gastro-intestinal, les premiers symptômes apparaissant entre 10 et 20 minutes après ingestion $(1,2)$. Le métabolisme, étudié chez l'animal, est essentiellement hépatique, par l'intermédiaire des cytochromes P450 (3). $70 \%$ à $90 \%$ de la dose administrée est métabolisé, le reste étant éliminé sous forme inchangée au niveau urinaire (4). Sa demi-vie plasmatique est comprise entre 10 et 16 heures (4-6). Elle agit par un mécanisme d'antagonisme compétitif de la glycine, neurotransmetteur inhibiteur, au niveau des récepteurs post-synaptiques des motoneurones (7). Il en résulte une hyperexcitabilité neuronale conduisant à une hyperactivité musculaire. Sur un plan clinique, on constate une hypertonie musculaire avec convulsions généralisées à la moindre stimulation sensorielle. Cette hypertonie prédomine aux muscles extenseurs, avec une attitude caractéristique en opisthotonos (8). Le risque vital est lié aux spasmes des muscles thoraciques et de la glotte entraînant une anoxie précoce et un arrêt cardiorespiratoire (1). Durant les crises convulsives, les patients sont habituellement conscients, permettant ainsi un diagnostic différentiel avec les autres phénomènes convulsifs comme l'épilepsie, le tétanos, les méningites et autres overdoses aux phénothiazines.

La strychnine, hautement toxique pour l'homme, était utilisée auparavant comme raticide mais n'est plus employée en France depuis les arrêtés de 1982 (9). Les empoisonnements à la strychnine étaient fréquents au siècle dernier. Aujourd'hui, bien qu'elles soient rares, les intoxications à la strychnine peuvent néanmoins survenir en raison notamment de la persistance de réserves de strychnine dans les régions rurales et de la présence de strychnine comme adultérant dans certaines poudres d'héroïne (10).

D'après la littérature $(4,11,12)$, des concentrations sanguines inférieures à $2 \mathrm{mg} / \mathrm{l}$ sont toxiques mais non mortelles, des concentrations comprises entre 2 et 10 $\mathrm{mg} / \mathrm{l}$ peuvent être mortelles et des concentrations supérieures à $10 \mathrm{mg} / 1$ sont toujours mortelles excepté un cas non mortel avec une concentration sanguine de $22 \mathrm{mg} / \mathrm{l}$ (13). Les doses habituellement létales de strychnine sont comprises entre 30 et $100 \mathrm{mg}$ (11).

Pour le dosage de la strychnine, plusieurs méthodes analytiques ont été publiées dans la littérature : chromatographie sur couche mince (14), chromatographie phase gazeuse couplée à la spectrométrie de masse (CPG/SM) $(4,12)$ ou à une spectrométrie de masse en tandem (CPG/SM/SM) (15), chromatographie liquide haute performance (CLHP) (16-18). Toutes ces techniques nécessitent au moins $1 \mathrm{ml}$ d'échantillon. Aucune méthode utilisant la chromatographie liquide haute performance couplée à une détection par barrette de diodes (CLHP/BD) n'a été décrite. Dans le cadre d'une affaire médico-légale pour laquelle une faible quantité de sang était disponible, nous avons développé une méthode de dosage de la strychnine par CLHP/BD ne nécessitant que $0,1 \mathrm{ml}$ d'échantillon.
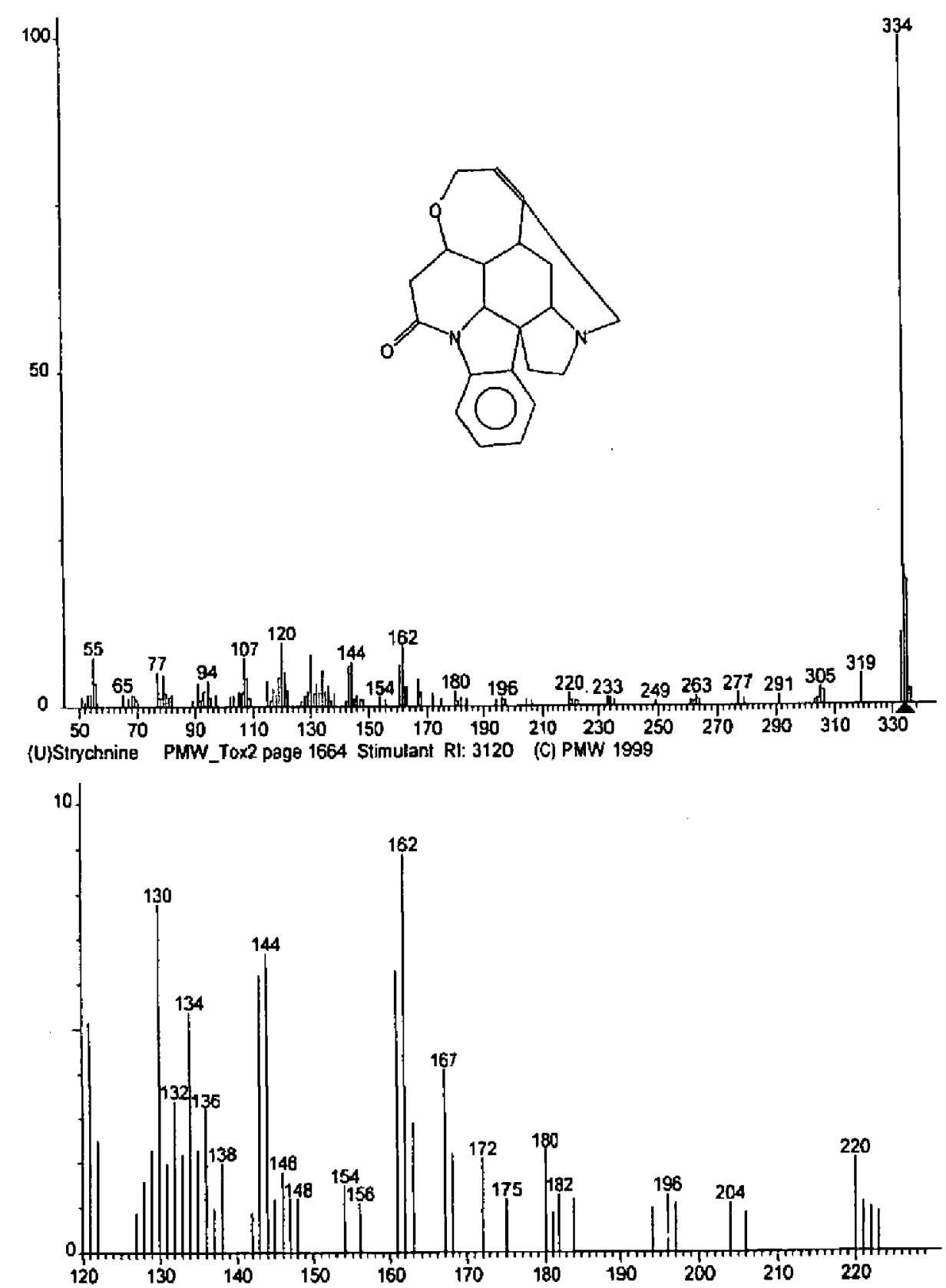

Figure 1 : Structure et spectre de masse de la strychnine.

\section{Cas clinique}

Un homme de 40 ans, M. D.G. est retrouvé mort chez lui. Une cannette de bière contenant seulement $0,5 \mathrm{ml}$ de liquide a été retrouvée à ses côtés. Aucun élément anormal n'ayant été constaté au cours de la levée de corps, l'autopsie n'a pas été demandée. Un échantillon de sang (environ $7 \mathrm{ml}$ ) et du liquide gastrique ont été 
prélevés par aspiration. Ces deux prélèvements ainsi que la cannette de bière ont ensuite été portés au laboratoire de Toxicologie. Un screening par CPG/SM et par CLHP/BD a été réalisé ainsi qu'une recherche de médicaments psychotropes (benzodiazépines, barbituriques, antidépresseurs et neuroleptiques) par des méthodes spécifiques utilisant la CPG/SM et la CLHP/BD. Une technique CPG/SM a été utilisée pour la recherche et le dosage de stupéfiants (opiacés, cocaïne, amphétamines, cannabis, méthadone et buprénorphine). L'alcool a été mesuré par méthode CPG couplée à une détection par ionisation de flamme.

\section{Matériel et méthodes Réactifs}

Tous les solvants utilisés sont de qualité chromatographique. La strychnine provient de Sigma (Paris, France). La chloroquine utilisée comme étalon interne est un don de Astra-Zeneca (Rueil, France). L'hydroxyde de sodium, le sodium dihydrogéno-phosphate $\left(\mathrm{NaH}_{2} \mathrm{PO}_{4}\right)$, le diéthyléther, le dichlorométhane et l'acide orthophosphorique proviennent de Sigma (Paris, France). L'acétonitrile et le méthanol ont été obtenus respectivement chez Riedel De Haën et Prolabo (Paris, France). Le tampon phosphate $20 \mathrm{mM}$ a été préparé dans de l'eau purifiée (système de purification d'eau AFS 200, Millipore, Molsheim, France) ajustée à pH 3,8 avec de l'acide orthophosphorique $10 \%$. Ce tampon est filtré préalablement à son utilisation à travers un filtre de $0,45 \mu \mathrm{m}$. Le plasma humain exempt de drogue a été obtenu à partir de l'établissement français du sang (Le Chesnay, France).

La solution stock de strychnine a été préparée à $1 \mathrm{~g} / \mathrm{l}$ dans du méthanol. Deux solutions de travail (à 100 $\mathrm{mg} / \mathrm{l}$ et à $10 \mathrm{mg} / \mathrm{l}$ ) ont été préparées en diluant la solution stock dans du méthanol. Les solutions stock et de travail de chloroquine (étalon interne) ont été préparées dans du méthanol respectivement à $1 \mathrm{~g} / 1$ et $10 \mathrm{mg} / \mathrm{l}$. Les solutions stocks et de travail sont conservées à $-20^{\circ} \mathrm{C}$ pendant 6 mois et 3 mois respectivement.

\section{Screening par CLHP/BD et CPG/SM}

Brièvement, une extraction en milieu acide et en milieu basique est réalisée par le mélange dichlorométhane/éther/hexane/alcool isoamylique (60/100/40/1). Les extraits organiques sont mélangés, évaporés puis repris par $50 \mu \mathrm{l}$ de méthanol. Deux $\mu \mathrm{l}$ de cette solution méthanolique sont injectés dans la CPG/SM. Le reste est évaporé et repris par $100 \mu \mathrm{l}$ d'un mélange tampon phosphate $20 \mathrm{mM}$ (pH 3,8)/acétonitrile (70/30). Cinquante $\mu$ l sont injectés dans la CLHP/BD.

\section{Préparation des standards et des contrôles de qualité}

Des volumes appropriés de solution de travail de strychnine sont ajoutés à $0,1 \mathrm{ml}$ de plasma humain sans drogue afin d'obtenir les concentrations finales de strychnine de 1, 2, 5, 10 et $25 \mathrm{mg} / \mathrm{l}$.

Un contrôle de qualité a été préparé avec des solutions indépendantes de manière à contenir $5 \mathrm{mg} / \mathrm{l}$ de strychnine.

\section{Préparation des échantillons}

Un volume de $0,1 \mathrm{ml}$ d'échantillon, de contrôle de qualité ou de standard est extrait par $2 \mathrm{ml} \mathrm{d}$ 'un solvant d'extraction éther-dichlorométhane ( $75: 25$, v/v) en milieu alcalin $(\mathrm{NaOH} 1 \mathrm{M}, 25 \mu \mathrm{l})$ après ajout de $50 \mu \mathrm{l}$ de chloroquine (E.I. à $10 \mathrm{mg} / \mathrm{l}$ ). Après 10 minutes d'agitation rotative et 5 minutes de centrifugation à $3000 \mathrm{tr} / \mathrm{min}$, la totalité de la phase organique est récupérée, évaporée et le résidu repris par $100 \mu \mathrm{l}$ d'un mélange tampon phosphate $20 \mathrm{mM} \mathrm{pH} 3,8$ /acétonitrile $(70: 30, v / v)$. Soixante $\mu \mathrm{l}$ sont injectés dans le système chromatographique. Ce système est constitué d'une pompe quaternaire P1000XR Spectra System, d'un injecteur à volume fixe : $100 \mu \mathrm{l}$ (Thermoquest, Les Ulis, France) couplé à un injecteur automatique (AS 3000 Spectra System), d'une colonne HyPurity C18 ThermoHypersil ( $250 \mathrm{~mm} \times 4,6 \mathrm{~mm}$ ) et d'une précolonne (C18, $5 \mu \mathrm{m}, 10 \mathrm{~mm} \times 4,4 \mathrm{~mm}$ I.D., ThermoHypersil) maintenues à $35^{\circ} \mathrm{C}$, et d'un détecteur par barrette de diodes UV 6000 (Thermoquest, Les Ulis, France). La quantification des molécules est réalisée à $254 \mathrm{~nm}$. L'élution est réalisée par un tampon phosphate $20 \mathrm{mM} \mathrm{pH} 3,8$ et un gradient linéaire d'acétonitrile de $10-40 \%$ en 9 minutes à un débit de 1 $\mathrm{ml} / \mathrm{minute}$

\section{Validation de la méthode}

Pour l'étude de linéarité, 6 courbes de calibration ont été réalisées sur 3 jours. La quantification a été effectuée en calculant le ratio entre l'aire du pic de strychnine et l'aire du pic de la chloroquine à la même longueur d'onde $(254 \mathrm{~nm})$. La précision et la justesse de la méthode ont été effectuées sur 3 jours. Chaque jour, une gamme a été réalisée ainsi que 4 déterminations du contrôle de qualité, soit 12 contrôles de qualité analysés sur 3 jours. Les valeurs obtenues ont été analysées en utilisant l'analyse de variance (ANOVA), permettant de séparer les déviations standard intra-jour et interjour et par conséquent les coefficients de variation correspondants $(\mathrm{CV})$. Le coefficient de variation intra-jour prend en compte la variabilité des 4 déterminations chaque jour pendant 3 jours, tandis que le coefficient de variation inter-jour s'intéresse à la variabilité du jour de 
l'analyse. La justesse a été déterminée en comparant la moyenne de la concentration calculée avec la concentration cible du contrôle de qualité. La limite de détection (LOD) est définie comme étant la concentration minimale de strychnine pouvant être détectée avec un rapport signal sur bruit de fond supérieur à $5: 1$, et la limite de quantification (LOQ) comme étant le premier point de gamme pour lequel les $\mathrm{CV}$ sont inférieurs à $20 \%$ et la justesse supérieure à $90 \%$.

Un rendement d'extraction a été déterminé pour l'ensemble des 12 contrôles de qualité étudiés. Ce rendement d'extraction a été déterminé en comparant les moyennes des aires des pics de strychnine obtenues à partir des contrôles de qualité à celle obtenue à partir de 5 standards de référence non extraits préparés à la même concentration $(5 \mathrm{mg} / \mathrm{l})$.

\section{Résultats}

\section{Validation de la méthode}

Les pics de strychnine et de chloroquine sont complètement résolus. Aucune interférence par des substances endogènes n'est observée. Les temps de rétention de la chloroquine et de la strychnine sont respectivement de 7,4 et 8,3 min. L'élution complète est obtenue en moins de $9 \mathrm{~min}$. Les courbes de calibration sont linéaires pour l'échelle comprise entre 1 et $25 \mathrm{mg} / \mathrm{l}$ (tableau I), avec une LOQ à $1 \mathrm{mg} / \mathrm{l}$. Le rendement d'extraction est de $94,1 \%$; tous les critères de validation sont corrects avec un coefficient de variation intra-essai inférieur à $6 \%$, un coefficient de variation inter-essai inférieur à $11 \%$ et des justesses inter-essai et intra-essai supérieures à $95 \%$ (tableau II). La limite de détection (LOD) est de $0,06 \mathrm{mg} / \mathrm{l}$. La sélectivité de la méthode a été étudiée vis à vis de certaines molécules fréquemment rencontrées en toxicologie : il n'y a pas d'interférence avec le nordiazépam, le diazépam (utilisé dans le traitement de l'intoxication à la strychnine), la clomipramine, l'imipramine, la nortryptiline, la cyamémazine et l'alimémazine.

\section{Résultats des analyses toxicologiques}

Les recherches spécifiques de médicaments psychotropes et de stupéfiants se sont révélées négatives. Il n'a
Tableau I : Linéarité, limite de quantification. Les courbes de calibration ont été obtenues grâce à 6 déterminations sur 3 jours.

\begin{tabular}{|c|c|c|c|c|c|}
\hline & \multicolumn{5}{|c|}{ Concentrations théoriques (mg/) } \\
\hline & 1 & 2 & 5 & 10 & 25 \\
\hline $\begin{array}{l}\text { Concentrations } \\
\text { mesurées (mg/) }\end{array}$ & 1,04 & 1,85 & 4,72 & 10,8 & 24,2 \\
\hline $\mathrm{CV} \%$ & 14,5 & 10,6 & 9,64 & 9,15 & 7,35 \\
\hline Biais \% & 4 & $-7,5$ & $-5,6$ & 8 & $-3,2$ \\
\hline Pente & \multicolumn{5}{|c|}{$5,0,8$} \\
\hline Origine & \multicolumn{7}{|c|}{0,9991} \\
\hline $\mathrm{r}$ & \multicolumn{6}{|c|}{} \\
\hline
\end{tabular}

pas été mis en évidence d'alcool ni dans l'échantillon de sang de M. D.G., ni dans le liquide de la cannette de bière. Les screenings CPG/SM et CLHP/BD ont montré la présence de strychnine. En raison d'une très faible quantité de sang disponible, la présente méthode a été développée et appliquée au sang, au liquide gastrique et au liquide contenu dans la cannette de bière afin de quantifier la strychnine. Cette substance a été détectée dans les trois échantillons à notre disposition avec des concentrations respectives de $25 \mathrm{mg} / \mathrm{l}, 760$ $\mathrm{mg} / 1$ et $850 \mathrm{mg} / \mathrm{l}$. La figure 2 présente le chromatogramme d'un extrait de $0,1 \mathrm{ml}$ du contenu gastrique de M. D.G. (dilué au centième).

\section{Discussion}

A notre connaissance, la méthode de chromatographie liquide présentée ici est la première utilisant la détection par barrette de diodes. L'utilisation d'un tel détecteur augmente la spécificité de la méthode en permettant l'identification des composés inconnus en comparant leurs spectres UV avec ceux d'une librairie (19). La librairie utilisée ici est celle qui a été développée dans notre laboratoire et commercialisée par la société Thermo-finnigan. Pour permettre cette identification spectrale, nous avons choisi comme limite de détection la concentration minimale de la molécule pouvant être détectée avec un rapport signal sur bruit de fond supérieur à $5: 1$, même si la LOD est généralement définie comme étant la concentration minimale de la molécule

Tableau II : Justesse, précision et rendement d'extraction pour un contrôle de qualité de concentration 5 mg/l de strychnine analysé par analyse de variance pendant 3 jours.

\begin{tabular}{|c|c|c|c|c|c|}
\hline & $\begin{array}{c}\text { CV Intra-essai } \\
(\boldsymbol{\%})\end{array}$ & $\begin{array}{c}\text { Justesse Intra-essai } \\
(\boldsymbol{\%})\end{array}$ & $\begin{array}{c}\text { CV Inter-essai } \\
(\boldsymbol{\%})\end{array}$ & $\begin{array}{c}\text { Justesse Inter-essai } \\
(\boldsymbol{\%})\end{array}$ & $\begin{array}{c}\text { Rendement } \\
\text { d'extraction }\end{array}$ \\
\hline $\begin{array}{c}\text { Strychnine } \\
5 \mathrm{mg} / \mathrm{l}(\mathrm{n}=12)\end{array}$ & 5,9 & 95,5 & 10,8 & 95,5 & 94,1 \\
\hline
\end{tabular}




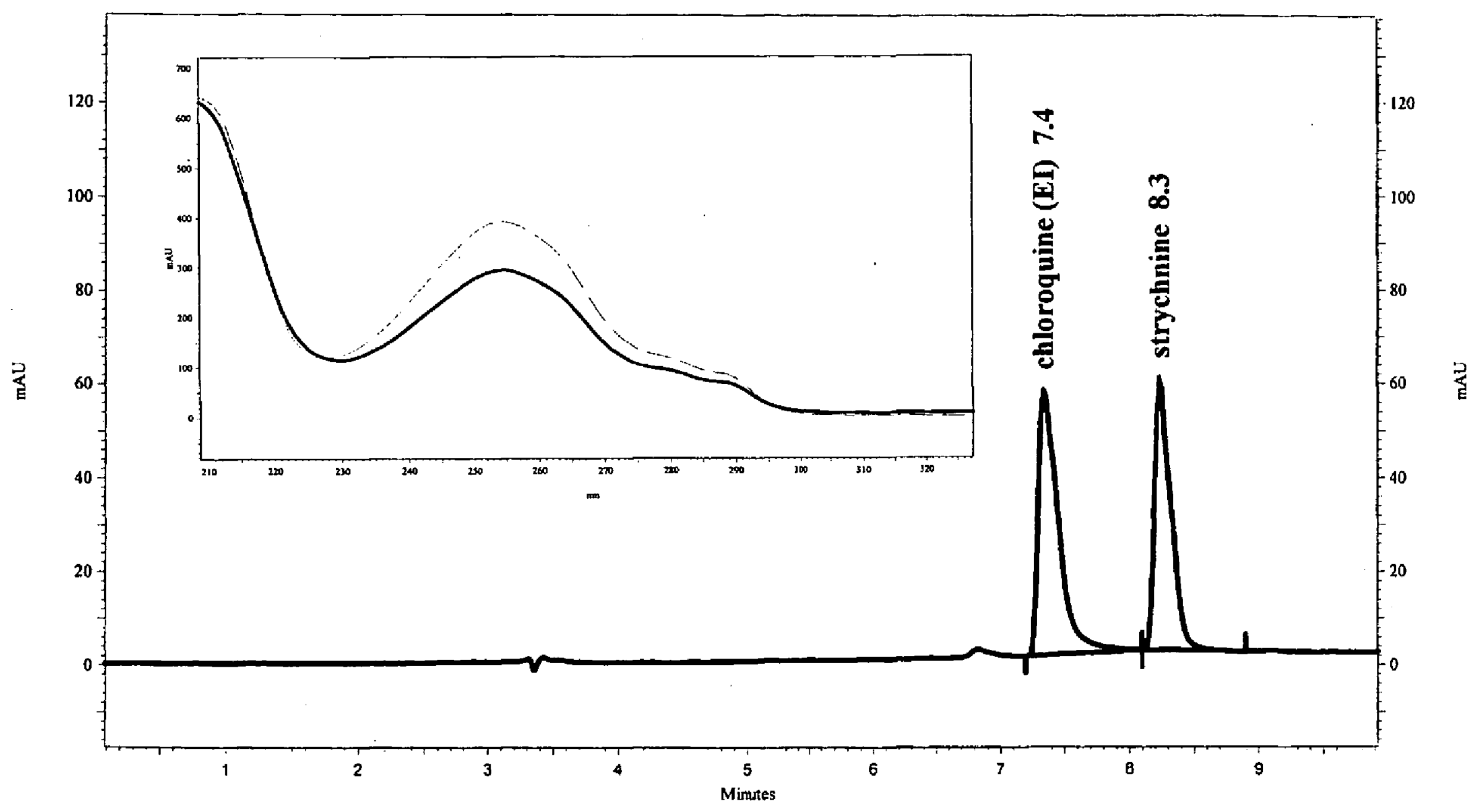

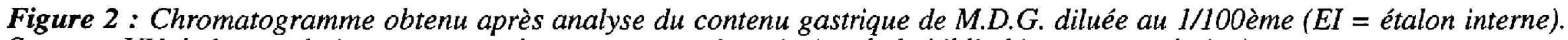
Spectres UV de la strychnine provenant du contenu gastrique (-) et de la bibliothèque spectrale (- -).

pouvant être détectée avec un rapport signal sur bruit de fond supérieur à $3: 1$. En raison d'une bonne sensibilité, cette méthode permet l'utilisation de seulement 0,1 $\mathrm{ml} \mathrm{d}$ 'échantillon tandis que toutes les méthodes précédemment publiées utilisaient au moins $1 \mathrm{ml}$ d'échantillon. Nous avons utilisé comme étalon interne la chloroquine car elle s'extrait parfaitement en milieu alcalin par le mélange d'extraction utilisé dans cette méthode et présente une bonne absorption à $254 \mathrm{~nm}$. Par ailleurs, il s'agit d'une molécule rarement rencontrée en pratique courante dans les échantillons de toxicologie. La validation de cette méthode a été réalisée dans le plasma car elle peut être appliquée à des échantillons provenant de sujets vivants. Chez les patients décédés, l'obtention de plasma s'avérant impossible, la méthode peut également s'appliquer au sang, la strychnine et la chloroquine présentant des rendements d'extraction similaires dans ces deux milieux (de l'ordre de $95 \%$ dans les deux cas).

La concentration de strychnine décelée dans le sang (25 mg/l) de M. D.G. était mortelle en accord avec les intoxications à la strychnine publiées dans la littérature $(4,11,12)$. Les concentrations similaires de strychnine dans le liquide gastrique et dans la cannette de bière sont en faveur d'une dissolution préalable de la strychnine dans la cannette préalablement vidée de son conte$\mathrm{nu}$, suivi de son absorption. N'ayant pas eu connaissance du volume total de liquide gastrique, la quantité de strychnine encore présente dans ce liquide n'a pu être calculée. En supposant que la cannette de bière était pleine, la quantité de strychnine ingérée serait de l'ordre de $280 \mathrm{mg}$, les doses minimales létales en l'absence de tout traitement variant selon les auteurs entre 30 et $100 \mathrm{mg}$ (11). Les investigations policières ont conclu à un suicide. La source de strychnine demeure un mystère compte tenu de la difficulté à se procurer de la strychnine en France aujourd'hui.

\section{Conclusion}

A notre connaissance, cette méthode de dosage de la strychnine est la première utilisant la Chromatographie Liquide Haute Performance couplée à une détection par barrette de diodes et ne nécessitant que $0,1 \mathrm{ml}$ d'échantillon. Les bons critères de validation de cette méthode, sa simplicité et sa spécificité permettent son utilisation aussi bien en toxicologie médico-légale qu'en toxicologie hospitalière, notamment lors d'intoxication chez les enfants où l'échantillon est souvent réduit. Elle a permis de confirmer un cas d'intoxication à la strychnine pour lequel une concentration sanguine de $25 \mathrm{mg} / \mathrm{l}$ a été mesurée, concentration considérée comme létale selon la littérature. 


\section{Références}

1. Teitelbaum D.T, Ott J.E. Acute strychnine intoxication. Clin. Toxicol. $1970 ; 3: 267-73$.

2. Sgaragli G.P., Mannaioni P.F. Pharmacokinetic observation on a case of massive strychnine poisoning. $J$. Toxicol. Clin. Toxicol. $1973 ; 6: 533-40$.

3. Mishima M., Tanimoto Y., Oguri Z., Yoshimura H. Metabolism of strychnine in vitro. Drug Metab. Dispos. $1985 ; 13: 716-21$.

4. Palatnick W., Meatherall R., Sitar D., Tenenbein M. Toxicokinetics of acute strychnine poisoning. J. Toxicol. Clin. Toxicol. $1997 ; 35: 617-20$.

5. Wood D.M., Webster E., Martinez D., Dargan P.I., Jones A.L. Case report : Survival after deliberate strychnine self-poisoning, with toxicokinetic data. Crit. Care, 2002 ; $6: 456-9$.

6. Edmunds M., Sheehan T.M.T., Van't Hoff W. Strychnine poisoning: clinical and toxicological observations on a non-fatal case. J. Toxicol. Clin. Toxicol., 1986 ; 24 : 245 55.

7. Probst A., Cortes R., Palacois J.M. The distribution of glycine receptors in the human brain. A light microscopic autoradiographic study using [3H] strychnine. Neuroscience, $1986 ; 17: 11-35$.

8. Smith B.A. Strychnine poisoning. J. Emerg. Med., $1990 ; 8: 321-5$

9. Arrêté du 24 février 1982 (JO du 10 mars 1982) relatif à l'emploi de la strychnine et de ses sels en agriculture.

10. Wijesekera A.R.L., Henry H.D., Ranasinghe P. The detection and estimation of (A) arsenic in opium, and (B) strychnine in opium and heroin, as a mean of identification of their respective sources. Forensic Sci. Int. 1988 ; $36: 193-209$.

11. Perper J.A. Fatal strychnine poisoning - A case report and review of the literature. J. Forensic Sci. 1985 ; 30 : 1248 55 .
12. Marques E.P., Gil F., Proença P., Monsanto P., Oliveira M.F., Castanheira A., Vieira D.N. Analytical method for the determination of strychnine in tissues by gas chromatography/mass spectrometry : two case reports. Forensic Sci. Int. $2000 ; 110: 145-52$.

13. François B., Cailloce D., Marquet P., Billy V., Lachatre G., Dumont D., Gay R., Strychnine poisoning : a report of 73 cases. Réan. Urg. 1996 ; 5 : 395-400.

14. De Saqui-Sannes P., Nups P., Le Bars P., Burgat V. Evaluation of an HPTLC Method for the Determination of Strychnine and Crimidine in Biological Samples. J. Anal. Toxicol. $1986 ; 20: 185-8$.

15. Rosano T.G., Hubbard J.D., Meola J.M., Swift T.A. Fatal strychnine poisoning : application of gas chromatography and tandem mass spectrometry. J. Anal. Toxicol. 2000 ; $24: 642-7$.

16.Egloff T., Niederwieser A., Pfister K., Otten A., Steinmann B., Steiner W., Gitzelmann P. A new high performance liquid chromatography (HPLC) method for the quantification of strychnine in urine and tissue extracts. J. Clin. Chem. Clin. Biochem. 1982 ; 20 : 203-6.

17. Alliot L., Bryant G., Guth P.S. Measurement of strychnine by high-performance liquid chromatography. J. Chromatogr. $1982 ; 232: 440-2$.

18. Hoogenboom J.J., Rammell C.G., Liquid chromatographic determination of strychnine in stomach contents. J. Assoc. Off. Anal. Chem. $1985 ; 68$ : 1131-3.

19. Gaillard Y., Pépin G. Use of high-performance liquid chromatography with photodiode-array UV detection for the creation of a 600-compound library. Application to forensic toxicology. J. Chromatogr. A. $1997 ; 763$ : 149 163. 